\title{
Percepción del alumnado sobre una Unidad Didáctica de enseñanza comprensiva de los juegos deportivos de invasión en Educación Física Students' perception of a Teaching Games for Understanding invasion games Unit in Physical Education Joan Úbeda-Colomer, Javier Monforte y José Devís-Devís Universitat de València (España)
}

Resumen. Este artículo examina las percepciones del alumnado acerca de una Unidad Didáctica (UD) de enseñanza comprensiva de los juegos deportivos de invasión. La UD se desarrolló durante ocho sesiones con la participación de 54 estudiantes de $3^{\circ}$ de ESO. Los datos se obtuvieron mediante un cuestionario ad hoc. Se calculó la media, la desviación típica y los porcentajes de los ítems como estadísticos descriptivos y se aplicó la prueba U de Mann Whitney con el fin de determinar las diferencias en función del sexo y la (no) práctica deportiva extraescolar. La pregunta abierta se analizó mediante un análisis de contenido y se calcularon los porcentajes de los aspectos emergentes. Los resultados revelan una valoración positiva de la UD. El alumnado destaca que se han explicado los principios tácticos de forma comprensible y la diversión durante la UD, así como la oportunidad de crear juegos propios y poder practicarlos. El alumnado que no practica deporte extraescolar percibe la UD más divertida que el alumnado que sí que lo hace. Además, las chicas encuentran la UD más divertida, interesante y comprensible que los chicos. Los resultados obtenidos ilustran las ventajas del modelo comprensivo para crear un entorno de práctica inclusivo y desarrollar una mejora en el pensamiento táctico que favorezca la satisfacción del aprendizaje. El trabajo concluye subrayando la importancia de tener en cuenta la percepción del alumnado para comprender y mejorar el modelo comprensivo de los juegos deportivos.

Palabras clave: enseñanza deportiva, juegos modificados, pensamiento del alumnado, TGfU, inclusión.

Abstract. This paper examines the student's perceptions of a Teaching Games for Understanding invasion games unit. The didactic unit (DU) was developed during eight lessons with the the participation of 54 students aged 14-16. Data were obtained through a questionnaire ad hoc. Mean, standard deviation and percentages from each item was calculated as descriptive statistics, and Mann-Whitney U test was carried out, in order to find the differences regarding sex and (non) extracurricular sport practice. The open question was processed through a content analysis and percentage from each emerging aspect was obtained. Results reveal a DU's positive evaluation. Students' highlight the understandable manner in which tactical principles are explained and their DU enjoying experience, as well as the opportunity to create and play their own games. Students that do not participate in extracurricular sports perceive funnier the DU than students that do participate. Furthermore, girls find the DU more enjoyable, interesting and understandable than boys. Results obtained illustrate the advantages of TGfU model to create an inclusive environment for practice, and the improvement in tactical thinking that support learning satisfaction. The study concludes highlighting the importance of considering students' perception for understanding and enhancing the TGfU model.

Keywords: sport teaching, modified games, students’ thinking, TGfU, inclusion.

\section{Introducción}

La aparición del modelo comprensivo de enseñanza de los juegos deportivos (Teaching Games for Understanding, TGfU) deriva de la insatisfacción docente que suscitaba la enseñanza deportiva centrada en los gestos técnicos (Bunker \& Thorpe, 1982). Las sesiones repetitivas y aburridas que alejaban al alumnado de los juegos deportivos, especialmente a las chicas y a las personas menos hábiles, provocaron la exploración de un modelo alternativo.Algunas de las ideas iniciales del nuevo modelo fueron el uso de pequeños juegos, la organización de la enseñanza a partir de los principios del juego y la adaptación del material para facilitar la ejecución del alumnado con menor habilidad. La principal aspiración consistía en hacer del alumnado el protagonista de su propia práctica, conseguir que comprendiera el juego para resolver los problemas tácticos que se le planteaban e, incluso, crear sus propios juegos (Bunker \& Thorpe, 1986). Estas ideas fueron extendiéndose a otros países de la mano de varios autores que tuvieron contacto con ellas (p.e. den Duyn, 1997; Devís-Devís, 1990; Devís-Devís \& Peiró-Velert, 1992, 2002; Werner, 1989; Liu, 1994) hasta consolidarse como un modelo de repercusión internacional, tal y como refleja su inclusión en manuales de educación física (EF) (p.e. Metzler, 2000) y la continuidad de congresos internacionales de enseñanza para la comprensión en los juegos deportivos (ver http://www.tgfu.org).

Desde una perspectiva internacional, se considera que el modelo comprensivo enfatiza el desarrollo cognitivo del alumnado a través de la resolución delos problemas tácticos generados en el juego y del desarrollo de estrategias colectivas (Light, 2008). Es decir, el alumnado debe desarrollar una comprensión que, ante cualquier situación de juego, le permita saber quétiene que hacer y cómo tiene que hacerlo, así como ser capaz de describir y explicar su actuación (Sánchez-Gómez, 2013).

Fecha recepción: 06-06-16. Fecha de aceptación: 15-11-16 José Devis Devis Jose.Devis@uv.es
Para ello, se utilizan los juegos modificados que permiten adaptar la dificultad al nivel de desarrollo del alumnado y poner el foco de atención en algún problema táctico (Devís-Devís \& Peiró-Velert, 1992; Dyson, Griffin \& Hastie, 2004). Esto es, mediante las modificaciones del espacio, las reglas y los equipamientos se representan o exageran distintas situaciones de juego para facilitar la comprensión contextual de los problemas tácticos en el alumnado (MacPhail, Kirk \& Griffin, 2008). En este contexto, el profesorado sirve de guía que ayuda a resolver los problemas tácticos del juego con la idea de reducir dicha ayuda progresivamente para que el alumnado gane autonomía y responsabilidad (Singleton, 2009; Light \& Fawns, 2003). El profesorado debe hacer preguntas durante el juego, pero dejándole jugar para recurrir posteriormente a pequeños debates o al uso de fichas que estimulen el pensamiento táctico, de tal forma que ayude al alumnado a analizar el juego y a buscar soluciones en la práctica (Bunker \& Thorpe, 1982; Devís-Devís \& Peiró-Velert, 1992; Pearson \& Webb, 2008; SánchezGómez, 2013).

Sin embargo, la popularidad académica del modelo y su inclusión en algunos currícula oficiales no parece corresponderse con una penetración equivalente en la EF escolar de la mayoría de países en los que se ha difundido (Almond, 2010; Stolz \& Pill, 2016). En muchos de ellos sigue siendo frecuente el énfasis en la ejecución técnica, de modo que la comprensión táctica y la reflexión sobre los aprendizajes suelen quedar en un segundo plano (p.e. Butler, 2005; Liu, 1997; Robles, Giménez \& Abad, 2011). El profesorado que ha aplicado el modelo muestra una percepción positiva del mismo porque lo considera inclusivo, divertido y relevante para el aprendizaje social y cognitivo del alumnado (DevísDevís, 1996; Light \& Butler, 2005; Light \& Tan, 2006; Wang \& Ha, 2013). Como señalan diversas investigaciones, el profesorado muestra interés en el modelo porque ofrece mayores oportunidades de participación a los estudiantes, mejora su motivación para aprender (Liu, 2002), favorece el intercambio de preguntas y respuestas y facilita el uso de discusiones sobre los juegos deportivos (Ha, Wang \& Collins, 2014). Sin embargo, también señalan dificultades en el desarrollo de 
ciertos juegos modificados, en definir estrategias de evaluación de la comprensión táctica (Devís-Devís, 1996) y en encontrar preguntas para favorecer la comprensión (Ruiz-Omeñaca \& Álvaro, 2015). Otros estudios recogen la inseguridad del profesorado con las modificaciones de los juegos (Ha, et al., 2014) y con su conocimiento sobre la enseñanza deportiva, así como la ansiedad, la confusión y la frustración que siente el profesorado con la aplicación del modelo (Díaz-Cueto, HernándezÁlvarez \& Castejón, 2010; Rossi, Fry, McNeill \& Tan, 2007).

Algunos trabajos se han ocupado de la opinión del alumnado sobre el modelo comprensivo y destacan la aceptación positiva, la mejora en el pensamiento táctico y la participación, así como las mejoras en la motivación, la diversión, la satisfacción y la competencia en los juegos deportivos (Chen \& Light, 2006; Fry, Tan McNeill \& Wright, 2010; Griffin, Oslin \& Mitchell, 1996; Jones, Marshall \& Peters, 2010; Light, 2003; Sánchez-Gómez, Devís-Devís \& Navarro, 2011). En un estudio cuasi-experimental se han encontrado cambios significativos en la percepción del alumnado en el aprendizaje y el esfuerzo después de una Unidad Didáctica (UD) de fútbol, independientemente del nivel de habilidad, aunque se han observado diferencias por sexo en la percepción del aprendizaje (Harvey, Wegis, Beets, Bryan, Massa-González \& van der Mars, 2009). También hay estudios que se ocupan de las dificultades o problemas que percibe el alumnado como, por ejemplo, el rechazo que muestran quienes tienen gran conocimiento deportivo previo y no entienden el sentido de las modificaciones en los juegos (SánchezGómez, 2013). Otros se refieren a la desmotivación que manifiestan cuando se dedica mucho tiempo a responder preguntas del profesorado y poco a jugar (Fry et al., 2010) o a la monopolización de las discusiones e intercambio de preguntas y respuestas del alumnado con más conocimiento deportivo o recursos comunicativos (Sánchez-Gómez, 2013). En algunas investigaciones se ha observado una separación entre la teoría y la práctica de la comprensión (Devís-Devís, 1996). Es decir, como apuntaba el alumnado en el estudio de Sánchez-Gómez (2013) «vemos [comprendemos] las cosas, pero no las podemos hacer» (p.385). Es la ‘brecha epistemológica’ a la que se refiere Light (2008) y que puede ser debida al predominio de estudios todavía de corta duración en las intervenciones, aunque algunos ya alcanzan un curso escolar. Por otra parte, se han observado diferencias en la comprensión según el sexo, atendiendo al conocimiento disponible en la cultura deportiva, ya que las chicas muestran más dificultades en los juegos de cancha dividida y, sobre todo, en los juegos de invasión que los chicos (Devís-Devís, 1996). También se ha encontrado que las chicas prefieren juegos que aumenten la participación, la cooperación y un esfuerzo menor, mientras que los chicos se decantan por los que implican mayores desafíos físicos y de habilidad (Gutiérrez \& García-López, 2012; SánchezGómez, et al., 2011).

La mayoría de estos estudios se desarrollan dentro de la tradición mediacional en la investigación sobre la enseñanza de la EF, caracterizada por el papel mediador que ejercen los procesos de pensamiento del profesorado y el alumnado entre la enseñanza y el aprendizaje (Hanke, 1987). Esto es, un tipo de investigación que enfatiza la visión cognitivista de la enseñanza y considera al profesorado y a los estudiantes como individuos activos que captan, procesan información y toman decisiones conscientes que finalizan en acciones que tienen sentido en el contexto interactivo de la enseñanza. Desde esta perspectiva, el aprendizaje del alumnado no depende solamente del tiempo de práctica y de su rendimiento motriz sino también de la percepción y significado que le atribuye a las experiencias de la enseñanza (Sicilia, 2002; Solmon, 2006). No obstante, esta tradición investigadora ha aparecido tarde en el estudio del modelo de enseñanza comprensiva de los juegos deportivos y, por lo tanto, las percepciones del alumnado se convierten en un ámbito de estudio emergente con poco recorrido todavía (Harvey \& Jarrett, 2014). Por tanto, se necesitan más estudios sobre el pensamiento del alumnado a la hora de aplicar el modelo TGFU, con el fin de comprenderlo y mejorarlo. La investigación centrada en el alumnado puede ayudar a identificar ventajas e inconvenientes de su uso, así como posibles aspectos a tener en cuenta para su desarrollo en la EF escolar. Por ello, el propósito de este artículo es el de conocer las percepciones de dos grupos de estudiantes de $3^{\circ}$ de la Educación Secundaria Obligatoria(ESO) sobre el desarrollo de una UD de juegos de invasión basada en el modelo comprensivo de enseñanza de los juegos deportivos.

\section{Metodología}

\section{La Unidad Didáctica: actividades, estrategias y evaluación}

Se diseñó una UD de juegos deportivos modificados de invasión. Es decir, juegos en los que se enfrentan dos equipos en un terreno de juego compartido para conseguir puntuar más veces que el contrario en la portería o meta contraria, mediante el desplazamiento de un móvil de juego. La UD tuvo una duración de ocho sesiones. Las primeras cinco las diseñó el profesorado, mientras que las tres siguientes se dedicaron a los juegos que el propio alumnado creó trabajando en pequeños grupos.

Los juegos se desarrollaron para favorecer el aprendizaje de objetivos tácticos básicos de ataque y defensa en dos grupos de $3^{\circ}$ de ESO de un centro de Educación Secundaria de la ciudad de Valencia. En concreto, los objetivos de ataque fueron el mantenimiento de la posesión del móvil, la progresión hacia la meta contraria y marcar en la meta contraria, mientras que los de defensa fueron evitar la progresión del contrario, recuperar la pelota y evitar ser marcado en la meta propia, en sintonía con lo que proponen Mitchell, Oslin y Griffin (2003).

En el desarrollo de la UD se realizaron modificaciones en diversas variables estructurales de los juegos de invasión, atendiendo a las consideraciones de Devís-Devís y Peiró-Velert (1992) y MéndezGiménez (1999). Por ejemplo, se utilizaron casi siempre canchas amplias que dejan más espacios a los jugadores para facilitar el juego de ataque. Cuando se quería influir sobre la verticalidad del juego se cambió a espacios más estrechos y a espacios anchos cuando se quería facilitar el juego por las bandas. Seutilizaron pelotas de goma-espuma y de voleibol no demasiado hinchadas, puesto que eran más fáciles de coger y reducían el nivel de demanda técnica. En ocasiones muy puntuales también se utilizaron pelotas de rugby para generar incertidumbre. La regla de parar (al tocar un adversario) o progresar en posesión de la pelota se introdujo en función de si se quería o no facilitar el pase y el desmarque. No se permitía robar el balón cuando el equipo contrario lo tenía en su poder, solo interceptar los pases, lo cual minimiza el contacto y la confrontación, al tiempo que facilita la participación del alumnado menos hábil. Por último, se utilizaron metas abiertas y grandes como superar la línea de fondo, ya que facilitan el objetivo ofensivo y son más estimulantes al principio. Poco a poco, se fueron introduciendo metas más pequeñas que generaban defensa en zona, como por ejemplo hacer llegar el balón a un compañero o compañera situada en la línea de fondo o depositar el balón en un aro. Casi siempre fueron metas fijas excepto en algunas ocasiones en las que se permitió desplazarse horizontalmente al jugador o jugadora que hacía de meta para generar una mayor riqueza en la estrategia de ataque. Predominó el juego con una meta por equipo, aunque a veces también se utilizaron dos metas en cada extremo para facilitar el objetivo en ataque y dificultar la defensa.

Para facilitar la comprensión y el pensamiento táctico del alumnado, además de las modificaciones, se recurrió a las intervenciones del profesorado a base de preguntas, siguiendo las orientaciones de Pearson y Webb (2008). Siempre se dejó jugar antes de intervenir para que el alumnado vivenciase los problemas que el juego planteaba y experimentase diferentes soluciones. Al finalizar el juego, se hacía una reflexión en gran grupo en la que el profesor formulaba diferentes preguntas que, de manera progresiva, guiaban al alumnado hacia la comprensión del problema táctico que se había querido trabajar durante el juego.Enocasiones seinterrumpía el juego en unmomento determinado para haceruna reflexión situada. También se empleó el debate en pequeños grupos, antes y después del juego, porque requería una argumentación más extensa y una mayor reflexión sobre los aspectos tácticos. Luego se reanudaba el juego para queel alumnado pudiese experimentar las nuevas soluciones debatidas. Además, el alumnado creó, en pequeños grupos, dos juegos modificados de invasión y los llevaron a la práctica durante 
las tres últimas sesiones. En estas sesiones se desarrolló la misma metodología que en las anteriores. Al final de la UD, se le entregó a cada alumno y alumna una ficha de trabajo con diez preguntas acerca de los aspectos tácticos más importantes de entre los que se habían trabajado en clase para entregarla cumplimentada una semana después.

Para recoger evidencias del aprendizaje del alumnado se recogió información sobre su participación en los juegos, la participación en los debates y las reflexiones, el trabajo grupal en la creación de los juegos de invasión y la ficha de trabajo individual. Cada uno de estos elementos representó el 25\% de la calificación final.

\section{Participantes e instrumento de recogida de datos}

La muestra estuvo compuesta por 54 estudiantes de $3^{\circ}$ de ESO, 24 chicos y 30 chicas, cuya edad oscilaba entre los 14 y los 16 años $(\mathrm{M}=14.54 ; \mathrm{DT}=.665)$. Para la recogida de datos, se diseñó un cuestionario ad hoc en el que se preguntaba al alumnado sobre determinados aspectos relativos a la UD para conocer su percepción acerca de la misma. El cuestionario contenía nueve ítems y el alumnado debía valorar su grado de acuerdo con cada uno de ellos en una escala Likert de 0 a 4 puntos en la que 0 era «Totalmente en desacuerdo» y 4 era «Totalmente de acuerdo». Los ítems que se incluyeron fueron los siguientes: «La UD me ha ayudado a comprender mejor los principios tácticos de los juegos de invasión»; «La UD ha sido divertida»; «Crear juegos en grupo y poder practicarlos ha sido interesante»; «Es interesante conocer y comprender los principios tácticos de los juegos de invasión»; «Los profesores han explicado los principios tácticos de forma comprensible»; «La dificultad de los juegos ha sido adecuada para que toda la clase participe sin problemas»; «Las preguntas que hacían los profesores ayudaban a descubrir los principios tácticos poco a poco»; «Mi forma de jugar ha ido mejorando a lo largo de la UD»; $y$ «Conocer los principios tácticos de los juegos de invasión permite jugar siendo consciente de lo que se hace». También se pidió al alumnado una valoración cuantitativa global, entre 0 y 10, de la UD. Además, se les preguntó, también en una escala de 0 a 10, su grado de interés por el deporte. Porúltimo, se incluyó una pregunta abierta en la que el alumnado debía expresar su opinión acerca de los aspectos positivos y negativos de la UD.

\section{Procedimiento}

En la última sesión de la UD se reservaron los 10 minutos finales para la cumplimentación del cuestionario. Se explicó al alumnado que el objetivo del mismo era conocer su opinión sobre la experiencia desarrollada para poder valorarla correctamente, así como detectar qué aspectos habían funcionado bien y cuáles requerían una mejora de cara a futuras aplicaciones del modelo de enseñanza comprensiva. La cumplimentación del cuestionario fue totalmente anónima y voluntaria.

\section{Análisis de los datos}

Para los análisis estadísticos se utilizó el software SPSS 22.0. En primer lugar, se realizó un análisis descriptivo para obtener la media y la desviación típica de los diferentes ítems, así como el porcentaje que obtenía cada categoría de respuesta en cada uno de ellos. Al no cumplirse el supuesto de normalidad para ninguna de las distribuciones, se utilizó la prueba U de Mann Whitney para muestras independientes y se determinaron las posibles diferencias en los distintos ítems en función de las variables «Sexo» y «Práctica de actividad físico-deportiva extraescolar». Para comprobar si existía relación entre el grado de interés por el deporte del alumnado, la nota de valoración otorgada a la UDy la edad se realizaron correlaciones de Spearman.

Por último, en la pregunta abierta en la que se pedía al alumnado que señalara lo que más y lo que menos le había gustado de la UD, se realizó un análisis de contenido con el fin de extraer los aspectos emergentes más importantes y se calculó el porcentaje de cada uno de ellos.

\section{Resultados}

En líneas generales, los resultados indican que la valoración que hace el alumnado de la UD desarrollada es altamente positiva. Como se observa en la tabla 1, el ítem que mayor puntuación obtiene es «Los profesores han explicado los principios tácticos de forma comprensible», con un 94.4\% del alumnado que manifiesta estar «De acuerdo» o «Totalmente de acuerdo» y una media de 3.61 puntos. A continuación, encontramos los ítems «La UD ha sido divertida», «La UD me ha ayudado a comprender mejor los principios tácticos de los juegos de invasión» $\mathrm{y}$ «La dificultad de los juegos ha sido adecuada para que toda la clase participe sin problemas», con los cuáles se muestran «De acuerdo» 0 «Totalmente de acuerdo» más del 80\% de los encuestados y que obtienen una media de 3.39, 3.22 y 3.19 respectivamente. Resultados similares obtienen los ítems «Crear juegos en grupo y poder practicarlos ha sido interesante», «Conocer los principios tácticos de los juegos de invasión permite jugar siendo consciente de lo que se hace» $\mathrm{y}$ «Las preguntas que hacían los profesores ayudaban a descubrir los principios tácticos poco a poco», con puntuaciones medias por encima de los 3 puntos y unos porcentajes de acuerdo que rondan o superan también el $80 \%$. Por otro lado, los ítems que obtienen las menores puntuaciones son «Es interesante conocer y comprender los principios tácticos de los juegos de invasión» $\mathrm{y}$ «Mi forma de jugar ha ido mejorando a lo largo de la UD», con unas medias de 2.81 y 2.78 respectivamente. En ambos casos, aunque el porcentaje de alumnado que se muestra «Totalmente en desacuerdo» $\mathrm{o}$ «En desacuerdo» sigue siendo enormemente bajo, existe un mayor número de alumnos y alumnas que se muestran neutrales al respecto, por lo que las puntuaciones quedan ligeramente por debajo de los 3 puntos.

En cuanto a las diferencias entre grupos, la prueba U de Mann Whitney muestra diferencias entre chicos y chicas en los ítems «La UD ha sido divertida» ( $U=461.5 ; \mathrm{p}<.05)$, «Crear juegos en grupo y poder practicarlos ha sido interesante» (U=499.5; $\mathrm{p}<$.01) y «Los profesores han explicado los principios tácticos de forma comprensible» ( $U=454.0$; $\mathrm{p}<.05$ ). En los tres casos, como se puede ver en la figura 1, las chicas dan puntuaciones más altas que los chicos, es decir, que las alumnas han considerado más divertida la UD, han considerado más interesante el hecho de poder crear juegos en grupo para practicarlos y se han mostrado más de acuerdo en que los profesores han explicado los principios tácticos de forma comprensible.

Además, también se encuentran diferencias entre el grupo que sí que practica actividad físico-deportiva extraescolar y el que no practica en el ítem «La UD ha sido divertida» $(\mathrm{U}=484.0$; $\mathrm{p}<.01)$. Como se observa en la figura 1, el alumnado que no practica actividad físicodeportiva extraescolar ha considerado la UD más divertida que el alumnado que sí que lo hace.

Las correlaciones de Spearman no muestran relaciones significativas entre el grado de interés por el deporte del alumnado ( $\mathrm{M}=7.53$; $\mathrm{DT}=1.77)$, la nota otorgada a la UD ( $\mathrm{M}=7.96 ; \mathrm{DT}=1.00)$ y la edad $(\mathrm{M}=14.54$; $\mathrm{DT}=.665)$.

Tabla 1.
Porcentajes en cada categoría de respuesta de los ítems con la media y desviación típica

\begin{tabular}{|c|c|c|c|c|c|c|c|}
\hline & $\begin{array}{c}\begin{array}{c}\text { Totalmente en } \\
\text { desacuerdo }\end{array}\end{array}$ & $\begin{array}{c}\text { En } \\
\text { desacuerdo }\end{array}$ & Indiferente & De acuerdo & $\begin{array}{l}\text { Totalmente } \\
\text { de acuerdo }\end{array}$ & M & SD \\
\hline La UD me ha ayudado a comprender mejor los PT de los JI & $0 \%$ & $1.9 \%$ & $11.1 \%$ & $50.0 \%$ & $37.0 \%$ & 3.22 & .718 \\
\hline La UD ha sido divertida & $0 \%$ & $3.7 \%$ & $14.8 \%$ & $25.9 \%$ & $55.6 \%$ & 3.39 & .811 \\
\hline Crear juegos en grupo y poder practicarlos ha sido interesante & $0 \%$ & $1.9 \%$ & $20.4 \%$ & $37.0 \%$ & $40.7 \%$ & 3.17 & .818 \\
\hline Es interesante conocer y comprender los PT de los JI & $0 \%$ & $1.9 \%$ & $33.3 \%$ & $46.3 \%$ & $18.5 \%$ & 2.81 & .754 \\
\hline Los profesores han explicado los PT de forma comprensible & $0 \%$ & $1.9 \%$ & $3.7 \%$ & $25.9 \%$ & $68.5 \%$ & 3.61 & .656 \\
\hline La dificultad de los juegos ha sido adecuada para que toda la clase participe sin problemas & $0 \%$ & $3.7 \%$ & $11.1 \%$ & $48.2 \%$ & $37.0 \%$ & 3.19 & .779 \\
\hline Las preguntas que hacían los profesores ayudaban a descubrir los PT poco a poco & $1.9 \%$ & $0 \%$ & $9.3 \%$ & $61.1 \%$ & $27.8 \%$ & 3.13 & .728 \\
\hline Mi forma de jugar ha ido mejorando a lo largo de la UD & $1.9 \%$ & $7.4 \%$ & $22.2 \%$ & $48.1 \%$ & $20.4 \%$ & 2.78 & .925 \\
\hline Conocer los PT de los JI permite jugar siendo consciente de lo que se hace & $0 \%$ & $5.6 \%$ & $11.1 \%$ & $46.3 \%$ & $37.0 \%$ & 3.15 & .833 \\
\hline
\end{tabular}




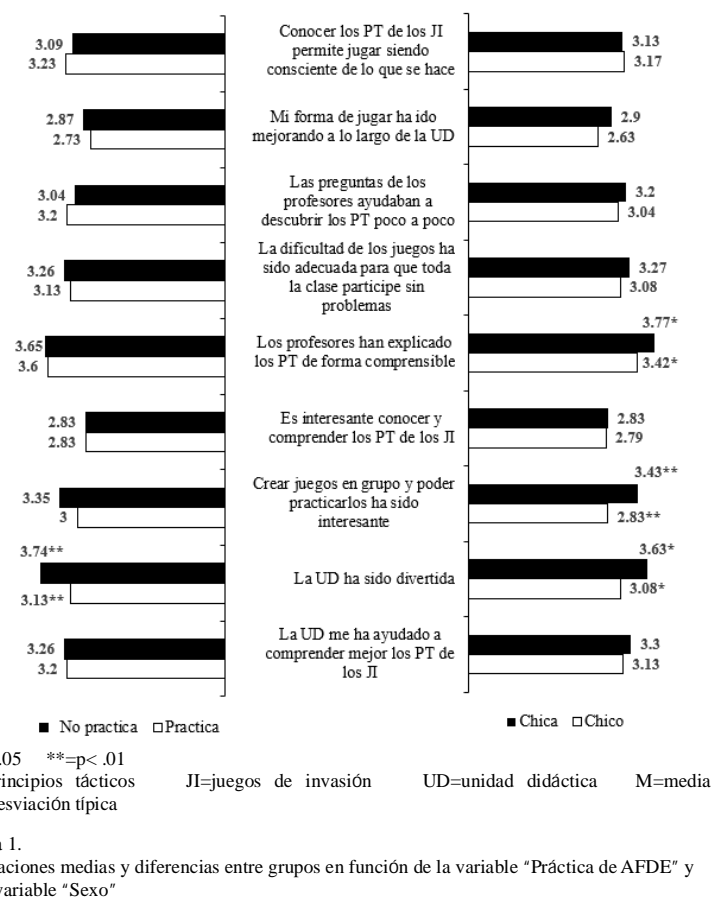

Entre los porcentajes de los aspectos derivados de la pregunta abierta destacan estas cuatro categorías principales: a) crear juegos propios y poder practicarlos (35.2\%); b) la diversión obtenida con los juegos (33.3\%); c) el aprendizaje y conocimiento de los principios tácticos (18.5\%); y d) la colaboración y el trabajo en equipo (13\%).

Entre los aspectos que menos les han gustado, aunque la frecuencia con la que se alude a éstos es mucho más baja, emergen otras cuatro categorías: a) las interrupciones en los juegos para preguntar y explicar aspectos sobre los principios tácticos (14.8\%); b) que algunos compañeros y compañeras pasan poco la pelota (3.7\%); c) la repetición de algunos juegos (3.7\%); y d) la poca participación de algunos compañeros y compañeras (3.7\%).

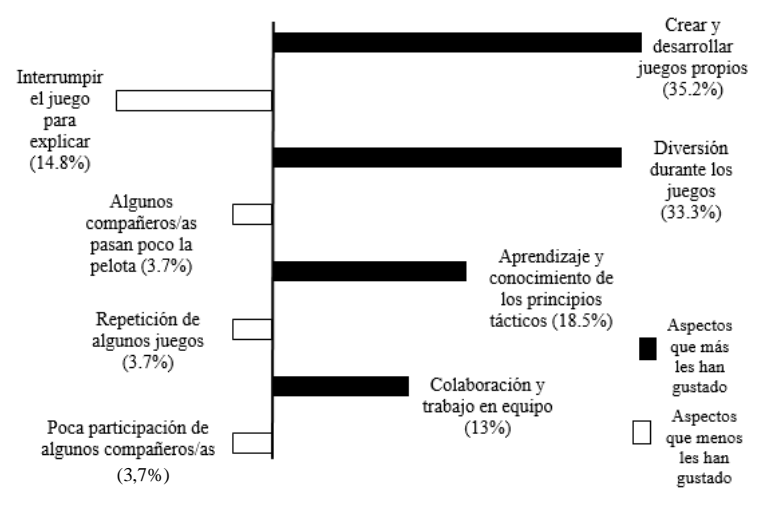

Figura 2.

Aspectos positivos y negativos emergentes y porcentaje del alumnado que alude a ellos

\section{Discusión}

En primer lugar, cabe señalar que la percepción del alumnado con respecto a la UD ha sido, en términos generales, muy positiva. Esto se observa tanto por las altas puntuaciones obtenidas en todos los ítems, como en los porcentajes de los aspectos derivados de la pregunta abierta, entre los que predominan los aspectos positivos sobre los negativos.

Uno de los resultados fundamentales en este trabajo es la amplia percepción de diversión y disfrute del alumnado durante la UD (95.5\% señala la categoría ‘de acuerdo’ o ‘totalmente de acuerdo’). La diversión también es uno de los aspectos que más estudiantes valoran en la pregunta abierta (33.3\%). Esto concuerda con los resultados de estudios previos que muestran la conexión entre el modelo de enseñanza comprensiva del deporte y el disfrute y diversión del alumnado (Chen \& Light, 2006; Fry, et al., 2010; Griffin, Oslin \& Mitchell, 1995; Light, 2003; Sánchez-Gómez, et al., 2011). Asimismo, se observan diferencias significativas entre chicos y chicas y entre quienes practican actividad física extra-escolar y quienes no lo hacen. Aunque en todos estos grupos se obtienen puntuaciones muy altas, en las chicas y en el alumnado que no practica actividad física extra-escolar los valores son significativamente mayores, superando los 3.5 puntos de media en ambos casos. Este resultado parece estar en sintonía con los hallazgos de Jones, Marshall y Peters (2010), ya que el modelo de enseñanza comprensiva del deporte generaba una mayor percepción de satisfacción en las chicas que en los chicos. Las altas puntuaciones de aquellos estudiantes que no practican actividad física extra-escolar pueden ser debidas a que su menor práctica, conocimiento deportivo o incluso una supuesta menor percepción de competencia no resulta un impedimento para el disfrute y satisfacción, contrariamente a lo que ocurre con un modelo tradicional. Esta argumentación es coherente con diversos trabajos que demuestran las ventajas del enfoque de enseñanza comprensiva del deporte para el alumnado con menores niveles de habilidad (Díaz-Cueto, et al, 2010) o depráctica física y menor percepción de competencia (Sánchez-Gómez, et al., 2011). Como el modelo TGFU no está centrado en la habilidad técnica, la motivación del alumnado menos hábil (o que tiene las características mencionadas anteriormente), suele mejorar en la toma de decisiones y en las relaciones sociales con sus iguales (Light, 2003).

Otro resultado destacable tiene que ver con la comprensión de los principios tácticos básicos de invasión, aspecto característico del modelo de enseñanza que nos ocupa. Las puntuaciones de varios de los ítems del cuestionario así lo indican. Así, por ejemplo, un alto porcentaje de estudiantes manifiestan haber mejorado en la comprensión de los principios tácticos (87\% se agrupa en las categorías 'de acuerdo' y 'totalmente de acuerdo') y señalan que los profesores han explicado los principios tácticos de forma comprensible (94.4\%), siendo esta percepción mayor entre las chicas que entre los chicos. Por contra, el $14.8 \%$ del alumnado señala en la pregunta abierta que no le ha gustado interrumpir el juego para hacer explicaciones y reflexiones dirigidas a la comprensión. En este sentido, Fry et al. (2010) encuentran que un tiempo excesivo de reflexión puede generar cierta insatisfacción, ya que el alumnado manifiesta no tener suficiente tiempo para disfrutar del juego. Sin embargo, debemos señalar que el $88.9 \%$ de los estudiantes se agrupan en las categorías ‘de acuerdo’ y ‘totalmente de acuerdo’ en el ítem que indica que las reflexiones les han ayudado a descubrir los principios tácticos poco a poco. Incluso existe un $68.5 \%$ que señala estar ‘de acuerdo' o ‘totalmente de acuerdo' en que ha ido mejorando su actuación en el juego durante el desarrollo de la UD. Estos resultados concuerdan con otros estudios que presentan mejoras en la toma de decisiones y el desempeño táctico del alumnado cuando utilizan el modelo TGFU (Chatzopoulos, Drakou, Kotzamanidou \& Tsorbatzoudis, 2006; Gray \& Sproule, 2011; Miller, Christensen, Eather, Gray, Sproule, Keay \& Lubans, 2016).

Los resultados también indican que una gran mayoría del alumnado percibe como adecuada la dificultad de los juegos para que toda la clase participe en los mismos (85.7\% responden 'de acuerdo' o 'totalmente de acuerdo’). La participación generalizada es uno de los logros ampliamente reconocidos, tanto por profesorado como por alumnado, en los distintos estudios centrados en el modelo comprensivo de enseñanza de los juegos deportivos (Liu, 2002; Fry et al., 2010; Light, 2003; Sánchez-Gómez, et al., 2011). Este aspecto resulta importante dado que, como señalan Allison y Thorpe (1997), el enfoque tradicional basado en la técnica pone de manifiesto y refuerza aún más la falta de habilidad de parte del alumnado, generando consecuencias tan negativas como la marginación de los menos hábiles. Ante la menor habilidad culturalmente aprendida de las chicas en juegos deportivos, especialmente de invasión, modelos instruccionales que impliquen su participación en las clases son clave para construir un currículum relevante para ellas (Ennis, 1999). En este estudio, los resultados obtenidos ponen de manifiesto las ventajas del modelo comprensivo para crear un entorno 
de práctica más inclusivo. No obstante, el 3.7\% del alumnado alerta en la pregunta abierta sobre la poca participación de algunos compañeros y compañeras. Por lo tanto, a pesar de ser un porcentaje muy bajo, se debe tener muy en cuenta y seguir trabajando para que todos y todas, sin excepción, puedan participar y disfrutar de la práctica físico-deportiva. Si no se cambian las malas experiencias en el contexto de la EF, el alumnado adolescente tendrá un mayor riesgo de ser personas inactivas en el futuro (Beltrán-Carrillo, Devís-Devís, Peiró-Velert, \& Brown, 2012; Martins, Marques, Rodrigues, Sarmento, Onofre \& Carreiro da Costa, 2016).

Otro resultado relevante es la buena valoración que realiza un amplio grupo de estudiantes acerca de ser ellos quienes creen y desarrollen sus propios juegos (78.4\% están ‘de acuerdo’ o ‘totalmente de acuerdo’). Este aspecto también es el que más positivamente destaca el alumnado (35.2\%) de entre todos los indicados en la pregunta abierta. En este sentido, como señalan Díaz-Cueto et al. (2010), el enfoque de enseñanza comprensiva permite implementar una perspectiva constructivista en EF. Desde esta perspectiva centrada en el proceso, el alumnado es sujeto activo de su propio aprendizaje, lo construye, mientras que el profesorado cobra un rol de facilitador. El profesorado va cediendo responsabilidad al alumnado a medida que gana experiencia, lo cual contribuye al desarrollo de un pensamiento autónomo (Tan, Chow \& Davids, 2012).

Finalmente, debemos señalar algunas limitaciones de este trabajo.A la luz de la literatura, la mejora en el juego y la toma de decisiones que manifiesta el alumnado no puede considerarse una evidencia firme, en parte, debido a la corta duración de la UD y, en parte, porque al tratarse de percepciones subjetivas, esa mejora podría estar sobreestimada. En este sentido, se podría haber empleado la técnica de observación sistemática, con el fin de contrastar la percepción del alumnado con la realidad del aula. Además, para dar más entidad a estetipo de experiencias, es recomendable llevar a cabo intervenciones más duraderas que introduzcan los juegos deportivos modificados de forma gradual.Aunque no es necesario seguir una progresión lineal entre los cuatro grandes grupos de juegos deportivos, es aconsejable empezar con juegos de blanco y diana, seguir con juegos de bate y campo y cancha dividida, y finalizar con juegos de invasión (Devís-Devís y Sánchez-Gómez, 1996). Esta progresión permite al alumnado comprender la naturaleza de los distintos juegos deportivos, incorporar y asentar el conocimiento práctico o ‘saber cómo', y desarrollar el pensamiento estratégico de manera más rica y completa. A pesar de las limitaciones señaladas, la principal fortaleza de este trabajo consiste en cubrir un vacío de conocimiento empírico sobre el pensamiento del alumnado en el marco del modelo TGfU, especialmente acentuado en el contexto español. Además, dada la naturaleza aplicada del estudio también contribuye a la mejora del modelo y a la promoción de sus posibilidades didácticas. Destaca especialmente la potencialidad del modelo para favorecer la inclusión de chicas y chicos menos hábiles, así como la implicación cognitiva del alumnado.

\section{Conclusiones}

Este estudio ha analizado la percepción del alumnado sobre una UD basada en la enseñanza comprensiva de los juegos deportivos de invasión. La valoración positiva del alumnado refleja una satisfacción por el trabajo realizado y confirma la viabilidad de este tipo de propuestas en EF. En sintonía con una perspectiva constructivista, el enfoque comprensivo ha estimulado al alumnado para tomar protagonismo en su propio aprendizaje. Por añadidura, ha hecho que el proceso de enseñanza sea más interesante, comprensible y divertido, en especial para las alumnas. También ha sido más divertido para el alumnado que no practica actividad física extra-escolar. Al diseñar la UD para que toda la clase pudiera participar, ha sido posible atender mejor las necesidades y preferencias de las personas menos hábiles, y asegurarse de que las mismas comprendían el porqué de lo que hacían y cómo lo hacían. De esta manera, el modelo comprensivo ha facilitado que las experiencias compartidas fueran disfrutadas por la gran mayoría del alumnado. Este trabajo ilustra el valor de adoptar un enfoque pedagógico comprensivo y la importancia de investigar la propia práctica para mejorar los procesos de enseñanza-aprendizaje en EF a partir del pensamiento del alumnado.

\section{Referencias}

Allison, S., \& Thorpe, R. (1997). A comparison of the effectiveness of two approaches to teaching games within physical education. A skills approach versus a games for understanding approach. The British Journal of Education, 28(3), 9-13.

Almond, L. (2010). Forward: Revisiting the TGfU brand. En J. Butler and L. Griffin (Eds.), More teaching games for understanding: Moving globally (pp. vii-x). Champaign: Human Kinetics.

Beltrán-Carrillo, V.; Devís-Devís, J.; Peiró-Velert, C. \& Brown, D. (2012). When physical activity participation promotes inactivity: Negative experiences of Spanish adolescents in Physical Education and sport. Youth and Society, 44(1), 3-27.

Bunker, D. \& Thorpe, R. (1982). A model for the teaching of games in secondary schools. Bulletin of Physical Education, 19, 5-8.

Bunker, D. \& Thorpe, R. (1986). Landmarks on our way to 'teaching for understanding.' En R. Thorpe, D. Bunker and L. Almond(Eds.), Rethinking games teaching (pp. 5-6). Loughborough: University of Technology, Loughborough.

Butler, J. (2005). 'TGfU Pet-agogy: Old dogs, new tricks and puppy school'. Physical Education and Sport Pedagogy 10(3), 225-40.

Castejón, F.J. (Coord.) (2010). Deporte y enseñanza comprensiva. Sevilla:Wanceulen.

Chatzopoulos, D., Drakou, A., Kotzamanidou, M., \& Tsorbatzoudis, H. (2006). Girls' soccer performance and motivation: Games vs technique approach. Perceptual and Motor Skills, 103(2), 463470.

Chen, S., \& Light, R. (2006). 'I thought I'd hate cricket but I love it!': Year six students' responses to Games Sense pedagogy. Change: Transformations in Education, 9(1), 49-58.

den Duyn, N. (1997). Game sense. Developing thinking players. Canberra: Australian Sports Comission.

Devís-Devís, J.(1990). Renovación pedagógica de la educación física: la enseñanza de los juegos deportivos II. Perspectivas de la Actividad Física y el Deporte, 5, 13-16.

Devís-Devís, J. (1996). Educación física, deporte y currículum. Investigación y desarrollo curricular. Madrid: Visor.

Devís-Devís, J. \& Peiró-Velert, C. (eds.) (1992). Nuevas perspectivas curriculares en Educación Física: la salud y los juegos modificados. Barcelona: Inde.

Devís-Devís, J. \& Peiró-Velert, C. (eds) (2002). Nuevas perspectivas curriculares en Educación Física: la salud y los juegos modificados. México DF: Secretaría de Educación Pública.

Devís-Devís, J. \& Sánchez-Gómez, R. (1996). La enseñanza alternativa de losjuegos deportivos: antecedentes, modelos actuales de iniciación y reflexiones finales. En J.A. Moreno y P.L. Rodríguez (Comps.), Aprendizaje deportivo (pp. 159-184). Murcia: Universidad de Murcia.

Díaz-Cueto, M., Hernández-Álvarez, J. L., \& Castejón, F. J. (2010). Teaching Games for Understanding to In-Service Physical Education Teachers: Rewards and Barriers Regarding the Changing Model of Teaching Sport. Journal of Teaching in Physical Education, 2010, 29, 378-398.

Dyson, B., Griffin, L. L., \& Hastie, P. (2004). Sport education, tactical games, and cooperative learning: Theoretical and pedagogical considerations. Quest, 56 (2), 226-240.

Ennis, C. (1999). Creating a culturally relevant curriculum for disengaged girls. Sport, Education and Society, 4(1), 31-50.

Fry, J.M., Tan, C.W.K., McNeill, M., \& Wright, S. (2010). Children's perspectives on conceptual games teaching: A value-adding experience. Physical Education and Sport Pedagogy, 15(2), 139158.

Gray, S., \& Sproule, J. (2011). Developing pupils’ performance in team 
invasion games. Physical Education and Sport Pedagogy, 16(1), $15-32$.

Griffin L., Oslin J.L. \& Mitchell S.A. (1995). An analysis of two instructional approaches to teaching net games. Research Quarterly for Exercise and Sport, 66, 65-66.

Gubacs, K. (2004). Implementing a tactical approach into a net/wall games unit. Journal of the Florida Association of Health, Physical Education, Recreation and Dance, 42(2), 8-10.

Gutiérrez, D. \& García-López L. M. (2012). Gender differences in game behaviour in invasion games. Physical Education and Sport Pedagogy, 17(3), 289-301

Ha, A.S., Wang, L. \& Collins, J. (2014). Perceptions of Hong Kong physical education teachers on Teaching Games for Understanding: Implications for continuing professional development. Educational Research Journal, 29(1-2), 91-110.

Hanke, U. (1987). Cognitive aspects of interaction in Physical Education. En G. T. Barrette, R. S. Feingold, C. Roger-Rees and M. Piéron (Eds.). Myths, models and methods in sport pedagogy (pp. 135141). Champaign: Human Kinetics.

Harvey, S., \& Jarrett, K. (2014).Areview of the game-centred approaches to teaching and coaching literature since 2006. Physical Education and Sport Pedagogy, 19(3), 278-300.

Harvey, S., Wegis, H., Beets, M.W., Bryan, R., Massa-González, A-N and van der Mars, H. (2009). Changes in student perceptions of their involvement in a multi-week TGfU unit of soccer: A pilot study. En T. Hopper, J. Butler and B. Storey (Eds.) TGfU Simply good pedagogy: Understanding a complex challenge (pp.101-114). Vancouver: Physical \& Health Education Canada,

Jones, R. J. A., Marshall, S., \& Peters, D. M. (2010). Can we play a game now? The intrinsic value of TGfU. European Journal of Physical and Health Education, 4(2), 57-63.

Light, R. (2003). The joy of learning: emotion and leaning in games through TGfU. Journal of Physical Education New Zealand,36(1), 93-108.

Light, R. (2008). Complex learning theory - its epistemology and its assumptions about learning: Implications for physical education. Journal of Teaching in Physical Education, 27, 21-37.

Light, R., \& Butler, J. (2005). A personal journey: TGfU teacher development in Australia and the USA. Physical Education and Sport Pedagogy, 10(3), 241-254.

Light, R., \& Fawns, R. (2003). Knowing the game: Integrating speech and action in games teaching through TGfU. Quest, 55(2), 161176.

Light, R., \& Tan, S. (2006). Culture, embodied experience and teachers' development of TGfU in Australia and Singapore. European Physical Education Review, 12(1), 99-117.

Liu, Y.K. (1994). Innovation of games teaching. CUHK Journal of Primary Education, 4 (2), 43-48.

Liu, Y.K. (1997). Games teaching: Changed or unchanged? Educational Research Journal, 12(1), 30-35.

Liu, Y.K. (2002). Games Teaching: Teaching Games for Understanding. Hong Kong: Hong Kong Institute of Education.

MacPhail, A., Kirk, D. \& Griffin, L. (2008). Throwing and Catching as Relational Skills in Game Play: Situated Learning in a Modified Game Unit. Journal of Teaching in Physical Education, 27, 100115.

Martins, J., Marques, A., Rodrigues, A., Sarmento, H., Onofre, M. \& Carreiro da Costa, F. (2016). Exploring the perspectives of physically active and inactive adolescents: how does physical education influence their lifestyles? Sport, Education and Society, DOI: $10.1080 / 13573322.2016 .1229290$

Méndez-Giménez,A.(1999). Efectos de la manipulación de las variables estructurales en el diseño de juegos modificados de invasión. Lecturas: Educación Física y Deportes. Revista Digital, 4(16). (Recuperado el 6 de abril de 2014, de http://www.efdeportes.com/ efd16/juegosm.htm)

Metzler, M.W. (2000). Instructional models for Physical Education. Boston: Allin and Bacon,

Miller, A., Christensen, E., Eather, N., Gray, S., Sproule, J., Keay, J., \& Lubans, D. (2016). Can physical education and physical activity outcomes be developed simultaneously using a game-centered approach? European Physical Education Review, 22(1), 113-133.

Mitchell, S.A.; Oslin, J.L. \& Griffin, L.L. (2003). Sport Foundations for elementary physical education. Champaing, IL: Human Kinetics.

Mosston, M., \& Ashworth, S. (1986). La enseñanza de la Educación Física. Barcelona: Hispano Europea.

Pearson, P. \& Webb, P. (2008) Developing effective questioning in Teaching Games for Understanding. Play to Educate. 1st Asia Pacific Sport in Education Conference: Ngunyawaiendi Yerthoappendi Play to Educate, 21 de enero. Sturt Campus, Adelaide: Flinders University. Recuperado el 8 de febrero de 2016 de: http:/ / $\mathrm{r}$ o . u o w . e d u . a u / c g i / viewcontent.cgi?article $=1054 \&$ context=edupapers

Robles, J., Giménez, F.J. \& Abad, M.T. (2011). Metodología utilizada en la enseñanza de los contenidos deportivos durante la E.S.O. Revista Internacional de Medicina y Ciencias de la Actividad Física y el Deporte, 10(41), 35-57.

Rossi, T., Fry, J.M., McNeill, M. \&. Tan C.W.K. (2007) The Games Concept Approach (GCA) as a mandated practice: views of Singaporean teachers. Sport, Education and Society, 12 (1), 93111.

Ruiz-Omeñaca, J.V., \& Álvaro, M. (2015). La puesta en práctica del modelo comprensivo en la iniciación deportiva. Dificultades y propuestas resolutivas. Tándem, 48, 1-9.

Sánchez-Gómez, R. (2013). La enseñanza para la comprensión de los juegos deportivos: un estudio de casos en enseñanza secundaria. Tesis doctoral: Universitat de València.

Sánchez-Gómez, R., Devís-Devís, J. \& Navarro-Adelantado, V.(2011). Los juegos y las modificaciones según el alumnado en el modelo comprensivo. Tándem: Didáctica de la Educación Física, 37, 2030.

Sánchez-Gómez, R., Devís-Devís, J. \& Navarro-Adelantado, V.(2014). El modelo Teaching Games for Understanding en el contexto internacional y español: una perspectiva histórica. Ágora para la educación física y el deporte, 16(3), 197-213.

Sicilia, A. (2002). La investigación sobre el pensamiento del alumnado. Una revisión desde la educación física. Revista de Educación,331, 577-613.

Singleton, E. (2009). From command to constructivism: Canadian secondary school physical education curriculum and teaching games for understanding. Curriculum Inquiry, 39(2), 321-342.

Solmon, M.A. (2006). Learner cognition. En D. Kirk, D. Maconald and M. O’Sullivan (Eds.), The Handbook in Physical Education (pp. 226-241). London: Sage Publications.

Stolz, S.A. \& Pill, S. (2016). Anarrative approach to exploring TGfUGS. Sport, Education and Society, 21(2) 239-261.

Tan, C. W. K., Chow, J. Y. \& Davids, K. (2012). 'How does TGfU work?': examining the relationship between learning design in TGfU and a nonlinear pedagogy. Physical education and sport pedagogy, 17(4), 331-348.

Thorpe, R., Bunker, D. \& Almond, L. (Eds.) (1986). Rethinking games teaching. Loughborough: University of Technology, Loughborough.

Underwood, G.L. (1988). Teaching and learning in physical education: A social psychological perspective. London: The Falmer Press.

Wang, L., \& Ha, A.S. (2013). Three groups of teachers' views, learning experiences, and understandings of teaching games for understanding. Physical Education and Sport Pedagogy, 18(3), 336350.

Werner, P. (1989). Teaching games: a tactical perspective. Journal of Physical Education, Recreation, and Dance, 60(3), 97-100. 